\title{
Radiotherapy in the Multidisciplinary Management of Merkel Cell Carcinoma
}

\author{
Michael D. Green, MD, PhD, and James A. Hayman, MD, MBA
}

\section{Abstract}

The management of Merkel cell carcinoma (MCC) requires multidisciplinary care for optimal patient outcomes. Radiotherapy (RT) is most commonly used as adjuvant therapy to improve locoregional control in patients with MCC who undergo surgery. Additionally, it can sometimes be used as definitive monotherapy for patients who decline or are not candidates for surgery and as palliative treatment in those with metastatic MCC. This article discusses the indications, treatment considerations, and recommended dose prescriptions for RT in the management of early- and advanced-stage disease. Considerable hope exists that immunotherapy advances will synergize with RT to further enhance clinical outcomes.

J Natl Compr Canc Netw 2018;16(6):776-781 doi: 10.6004/jnccn.2018.7045

Merkel cell carcinoma (MCC) is a relatively rare cutaneous malignancy, predominantly diagnosed in elderly individuals (aged $>70$ years), that is increasing in incidence as the population ages. ${ }^{1}$ Due to the rarity of this disease, there is a paucity of prospective randomized studies to guide clinical practice. This review explores the role of radiotherapy (RT) as part of the multimodality management of MCC and presents current areas of consensus and controversy.

MCC arises from epidermal neuroendocrine cells, which synapse with afferent neurons to enable tactile sensation in the skin. ${ }^{2}$ It classically presents as a benignappearing; small; firm; red, purple, or skin-colored; nontender papule or nodule on a sun-exposed surface in an elderly individual. ${ }^{3}$ Diagnosis is made histologically. It is in the family of small round basophilic tumors, but can be distinguished based on S-100-negative, TTF-1-negative, and CK20-positive immunohistochemical staining. ${ }^{4}$ The isolation of clonal polyomavirus integration in MCC has suggested a viral contribution to the development of this disease in a subset of patients. ${ }^{5}$ Although it can occur in young adults, the frequency of the disease

Department of Radiation Oncology, University of Michigan, Ann Arbor, Michigan.

Submitted January 25, 2018; accepted for publication May 7, 2018.

The authors have disclosed that they have no financial interests, increases markedly in patients aged $>70$ years, which has been attributed to the burden of chronic mutagenic UV exposure and age-associated diminished immunosurveillance. ${ }^{3,6}$ It is also more common in patients immunosuppressed for other reasons (eg, transplant recipients, chronic lymphocytic leukemia). Prognosis is strongly dependent on the extent of disease at initial presentation and immune status.

Staging reflects the extent of disease, with distinctions made for small $(<2 \mathrm{~cm})$ versus moderately sized $(>2$ $\mathrm{cm})$ localized disease, regional disease involving draining lymph nodes, and disseminated metastatic disease. ${ }^{7}$ A pooled patient-level meta-analysis has confirmed that sentinel lymph node biopsy (SLNB) has increased sensitivity in detecting regional disease compared with CT scans. ${ }^{8}$ National guidelines now recommend that suitable patients with clinically node-negative MCC undergo SLNB, and that those with clinically node-positive MCC undergo biopsy (eg, fine-needle aspiration, core, open) to confirm lymph node involvement. ${ }^{9}$ Although staging with CT and/or PET/CT is encouraged whenever metastatic or unresectable disease is suspected based

arrangements, affiliations, or commercial interests with the manufacturers of any products discussed in this article or their competitors.

Correspondence: James A. Hayman, MD, MBA, Department of Radiation Oncology, University of Michigan, 1500 East Medical Center Drive, Ann Arbor, MI 48109. Email: hayman@med.umich.edu 
on patients' signs and/or symptoms, it is also recommended for all patients with pathologic evidence of lymph node involvement. ${ }^{8,9}$ The treatment of MCC is dependent on disease extent.

\section{Localized Disease Management}

Surgery represents the primary treatment modality for the diagnosis and treatment of localized MCC in medically and technically operable patients. ${ }^{9}$ A National Cancer Database (NCDB) study examining US patients diagnosed from 1985 to 2005 indicated that approximately $50 \%$ present with stage I or II disease. ${ }^{10}$ In line with the infiltrative nature of neuroendocrine malignancies, historical series have reported local recurrence rates of $100 \%$ following excision with only $0.5-\mathrm{cm}$ margins. ${ }^{3,11}$ Therefore, a wider local excision of 1 to $2 \mathrm{~cm}$ circumferentially and down to the investing fascia is the recommended surgical approach.

A number of older, single-institution, retrospective studies have indicated that adjuvant RT to the primary site is beneficial in early-stage MCC. ${ }^{12-15} \mathrm{Up}$ to $40 \%$ of all patients will develop local recurrences after surgery even with wide margins, which can be used to justify the routine use of adjuvant RT to the primary site. ${ }^{16}$ In fact, the largest systematic review to date of 1,254 patients with MCC treated from 1966 to 2004 showed a statistically significant reduction in local (hazard ratio [HR], 0.27) and regional (HR, 0.34) recurrence among patients treated with combination RT and surgery compared with surgery alone. ${ }^{17}$ Moreover, in one NCDB study, the addition of RT to surgery in patients with stage I and II disease was associated with improved survival. ${ }^{18}$ Further, a retrospective evaluation from British Columbia has suggested that RT is highly effective for local control following subtotal resection or microscopically positive margins. ${ }^{19}$

In contrast, several series have suggested that local recurrences are less common in patients with very early-stage MCC treated with surgery alone. The University of Michigan examined a cohort of 104 patients with stage I MCC treated from 2006 to 2012 who achieved a gross total resection via a wide local excision with 1 - to 2-cm margins and an SLNB. With a median follow-up of 3 years, local recurrences occurred in $1 \%$, satellite recurrences in $1 \%$, and in-transit recurrences in 3.8\%. ${ }^{20}$ At Memorial Sloan
Kettering Cancer Center, only 13\% of patients with stage I MCC received adjuvant RT, and a local recurrence rate of $2 \%$ was reported. ${ }^{21}$ In summary, retrospective data suggest that adjuvant RT directed to the tumor bed is generally beneficial, but there appears to be a subset of patients with such favorable disease that its omission can be considered.

Medically inoperable patients, patients in whom surgery would result in unacceptable functional or cosmetic outcomes, or those who decline surgery may be candidates for definitive RT alone. Radiobiological studies have indicated that the disease is very radiosensitive. ${ }^{22}$ Single-institution studies have indicated that local control with RT monotherapy for localized disease can range from $75 \%$ to $100 \% .23-25$ RT is also effective for head and neck locations in which surgical excision can be challenging. ${ }^{26}$

\section{Primary Site RT Treatment Considerations}

Gross tumor volume (GTV) is defined as the gross primary lesion and involved nodes (if any). Radially, clinical tumor volume typically encompasses the tumor or tumor bed with a 3 - to $5-\mathrm{cm}$ margin, although smaller expansions of 2 to $3 \mathrm{~cm}$ are sometimes used, depending on anatomic boundaries and adjacent normal tissues (eg, for head and neck sites). The full thickness of the skin and underlying fascia should also be targeted (typically, 5-mm thickness) often using tissue-equivalent material, commonly referred to as bolus, to achieve an adequate skin dose. Depending on the location and immobilization used, a 0.5to $1-\mathrm{cm}$ planning target volume (PTV) expansion can be used (Figure 1). In the adjuvant setting, the prospective phase II TROG 96:07 trial prescribed 50 Gy in 25 fractions using 2D beam arrangements, ${ }^{27}$ whereas the prospective Groupe de Cancérologie Cutanée of the Société Française de Dermatologie trial advised using the same dose, but frequently patients also received an additional 10-Gy boost to the tumor bed..$^{28}$ In the setting of gross disease, Canadian data have suggested that cancer-specific survival is improved if $>50$ Gy is administered. ${ }^{29}$ RT can be delivered using a number of modalities, including photons, electrons, or brachytherapy. Photon plans can be delivered conformally using 3D and volumetric techniques. Electrons are often used for the primary site because of the superficial nature of the region that needs to be treated. Case reports have indicated 


\section{A}

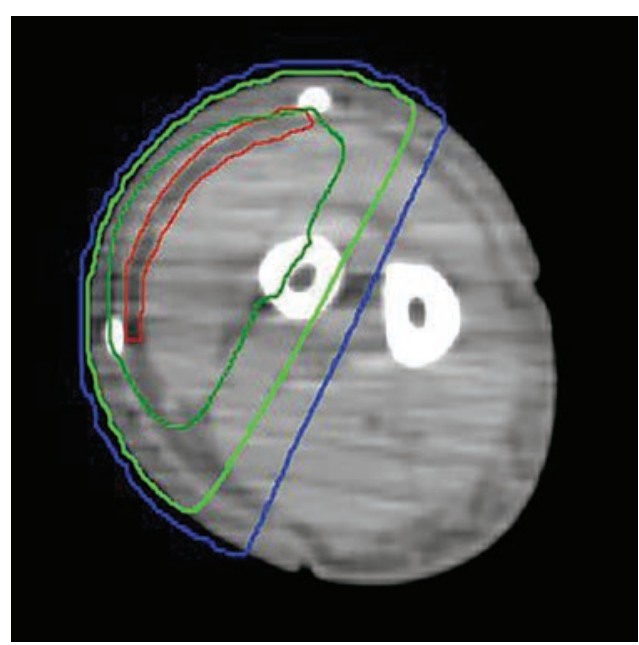

B

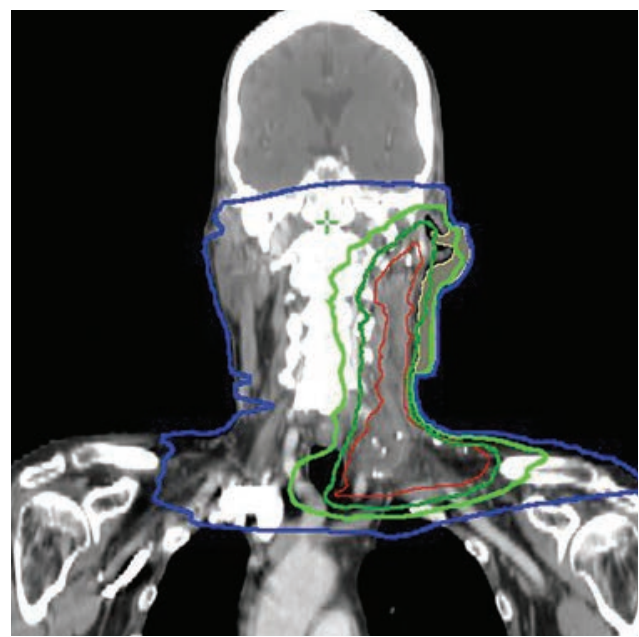

Figure 1. Primary site and regional nodal radiotherapy (RT) fields for Merkel cell carcinoma (MCC). (A) An oblique 3D beam arrangement with photon RT was used to adjuvantly treat the skin and subcutaneous tissues around the primary site (clinical tumor volume [CTV]: red) following wide local excision of a T2,NO MCC of the left forearm. Bolus was used to ensure full dose to the skin. 95\% (dark green), 50\% (green), and $10 \%$ (blue) isodose lines are shown. (B) Volumetric modulated arc therapy was used to conformally deliver adjuvant photon RT to a patient with stage IIIB MCC of the left cheek involving the parotid gland (CTV: red) following wide local excision and left neck dissection. Again, bolus (yellow) was used to ensure full dose to the skin around the primary site. 95\% (dark green), 50\% (green), and 10\% (blue) isodose lines are shown.

that brachytherapy can be effective in locations such as the lip, which are challenging to treat with external-beam RT. ${ }^{30}$ Using conventional fractionation of 1.8 to 2 Gy per treatment, the total radiation dose prescription for the primary site ranges from 50 to 56 Gy for negative surgical margins, 56 to 60 Gy for microscopically positive margins, and 60 to 66 Gy for gross disease (Table 1), although some believe that given the very radiosensitive nature of MCC, lower doses could also be used. ${ }^{31}$ Hypofractionated

\begin{tabular}{|c|c|c|}
\hline Site & Clinical Scenario & Recommended Dose \\
\hline \multicolumn{3}{|c|}{ Primary } \\
\hline & $\begin{array}{l}\text { Resected with wide margins } \\
(e g, 1-2 \mathrm{~cm}) \text { and small tumor size } \\
(<1-2 \mathrm{~cm})\end{array}$ & Consider observation \\
\hline & Resected with negative margins & $50-56$ Gy \\
\hline & $\begin{array}{l}\text { Resected with microscopically } \\
\text { positive margins }\end{array}$ & $56-60 \mathrm{~Gy}$ \\
\hline & $\begin{array}{l}\text { Resected with grossly positive } \\
\text { margins }\end{array}$ & $60-66$ Gy \\
\hline & $\begin{array}{l}\text { Not resected; definitive } \\
\text { radiotherapy }\end{array}$ & 60-66 Gy \\
\hline \multicolumn{3}{|c|}{ Nodal basin } \\
\hline & $\begin{array}{l}\text { Clinically node-negative with } \\
\text { SLNB negative }\end{array}$ & Observation \\
\hline & $\begin{array}{l}\text { Clinically node-negative without } \\
\text { SLNB or LN dissection }\end{array}$ & 46-50 Gy \\
\hline & $\begin{array}{l}\text { Clinically node-negative with } \\
\text { SLNB positive without LN } \\
\text { dissection }\end{array}$ & $50-56 \mathrm{~Gy}$ \\
\hline & $\begin{array}{l}\text { LN dissection with extracapsular } \\
\text { extension, multiple positive LNs }\end{array}$ & 50-60 Gy \\
\hline & $\begin{array}{l}\text { Clinically involved nodes without } \\
\text { SLNB or LN dissection }\end{array}$ & 60-66 Gy \\
\hline
\end{tabular}

Abbreviations: LN, lymph node; SLNB, sentinel lymph node basin.

regimens are also well tolerated in other cutaneous malignancies, ${ }^{32}$ and can be considered in patients in whom the delivery of conventionally fractionated RT is logistically challenging. For example, other options for gross disease include 50 Gy delivered in 20 fractions or 50 Gy delivered in 15 fractions. Additional clinical considerations that may influence the dose chosen within these ranges include the location, RT field size, and presence of adjacent critical normal structures.

\section{Regional Nodal Disease Management}

The risk of lymph node involvement is directly proportional to the size of the primary tumor. ${ }^{10}$ However, as noted, occult regional involvement can occur in $20 \%$ to $30 \%$ of patients even with $<2 \mathrm{~cm}$ primary lesions, and therefore SNLB is recommended in all patients who are clinically node-negative. ${ }^{14}$ Pathologic features based on the primary-site biopsy that predict SLNB positivity include increasing clinical tumor size, increasing tumor thickness, increasing mitotic rate, and infiltrative tumor growth. ${ }^{33}$ For patients with early-stage disease who are clinically node-neg- 
ative and decline or are not candidates for SLNB, treatment of the regional nodes with RT alone is supported by the results of the French randomized trial comparing adjuvant regional nodal RT versus observation in patients with stage I MCC following wide local excision and adjuvant RT to the tumor bed. ${ }^{28}$ Although the trial closed early due to poor accrual following the introduction of SLNB, the addition of nodal RT resulted in a significant improvement in regional recurrence ( $0 \%$ vs $17 \% ; P=.007$ ), although it was not associated with a difference in overall or progression-free survival. For patients with a negative SLNB, adjuvant nodal RT is not recommended. In patients with regional lymph node involvement, consensus guidelines favor lymph node dissection more strongly in fit patients with clinically apparent disease versus those with clinically node-negative, SLNB-positive disease, but definitive RT can also be considered in both settings. The advantage of further histologic characterization of disease burden afforded by surgery must be balanced against the potential morbidity of lymphadenectomy (eg, lower-extremity lymphedema following inguinal lymph node dissection). Multiple trials have evaluated the utility of adjuvant RT following complete surgical resection of nodal disease in patients with locally advanced melanoma, but no such data exist for MCC. ${ }^{32,34}$ If extracapsular extension or multiple lymph nodes are found to be involved, adjuvant RT is generally recommended out of concern for locoregional relapse.

Regional nodal disease is also very sensitive to RT. ${ }^{19}$ RT can be considered for the definitive or adjuvant treatment of regional lymph nodes. The University of Washington experience of 43 patients with node-positive MCC compared definitive RT for nodal management versus completion lymphadenectomy. ${ }^{35}$ With 18 months of follow-up, regional lymph node control was $100 \%$ in patients with subclinical microscopic disease treated with either RT or lymph node dissection, and was approximately 75\% (not statistically significantly different) for clinically involved nodes treated with either RT or lymph node dissection. In summary, RT offers excellent regional control in patients whose nodal disease burden has not been surgically addressed for technical or medical reasons.

\section{Regional Nodal RT Treatment Considerations}

The GTV and PTV definitions for regional disease are similar to those used in primary MCC. Clinical target volumes for regional nodes should encompass the entire nodal chain, and radial expansion beyond this volume are not typically used (Figure 1). ${ }^{18} \mathrm{~F}-\mathrm{FDG}-\mathrm{PET} / \mathrm{CT}$ can reveal occult disease and therefore can sometimes be used for more accurate target delination. ${ }^{36}$ In cases in which the primary sites and nodes are both being treated and the intervening distance is not too great, treatment of the intervening skin can be considered in hopes of decreasing the likelihood of developing in transit metastases. Our practice is to consider this if the intervening distance is $<20 \mathrm{~cm}$. The benefit of improved cutaneous control must be weighed against the potential for increased skin toxicity, especially in patients with head and neck cancer. Notably, TROG 96:07 used 2D beam arrangements and fields were set based on bony anatomy, not using cross-sectional delineation of target volumes. 3D and intensity-modulated RT can significantly reduce the dose received by organs at risk and improve the homogeneity of the radiation dose, which could limit acute skin and other late toxicities. ${ }^{37}$ Because of the deeper location of most nodal basins, photon RT is typically preferred. The radiation prescription typically ranges from total doses of 46 to $50 \mathrm{~Gy}$ for elective nodal radiation, 50 to 56 Gy for known microscopic nodal disease, and 60 to 66 Gy for gross disease (Table 1). ${ }^{31,38}$ NCDB analyses indicate that moderately lower doses of 45 to $50 \mathrm{~Gy}$ may be used for subclinical nodal disease..$^{39,40}$

\section{Role of Concurrent RT and Chemotherapy}

Single-institution data have repeatedly demonstrated that although RT can provide effective local control, up to $60 \%$ of patients treated definitively experience out-of-field relapses. ${ }^{24,25}$ This has led to treatment intensification studies in which concurrent chemotherapy was added to RT. The TROG 96:07 trial enrolled 53 patients with MCC with at least one of the following high-risk features: recurrence after local therapy, stage III disease, grossly positive surgical margins, tumors $>1 \mathrm{~cm}$, or an unidentified primary site. ${ }^{27}$ Patients received 50 Gy of $\mathrm{RT}$ in 25 fractions to the primary site and regional 
lymph nodes with a 3- to 5-cm margin, along with concurrent carboplatin and etoposide. Treatment was well tolerated aside from common acute skin toxicity (64\% grade $3 / 4)$. The 3-year overall survival, locoregional control, and distant control rates were $76 \%, 75 \%$, and $76 \%$, respectively. Multivariate analysis on a matched historical cohort did not identify receipt of chemotherapy as improving overall survival. ${ }^{41}$ The lack of benefit from cytotoxic chemotherapy has been further validated in NCDB studies. ${ }^{18}$ Therefore, despite the risk for systemic failures, adjuvant chemotherapy is not indicated in patients with locally advanced disease.

\section{Metastatic Disease Management}

The goal of treatment in patients with widely disseminated disease is to palliate symptoms. Locoregional techniques including surgery and RT can sometimes have significant therapeutic impact on achieving this goal. Palliation of bone metastases and other sites of extracranial metastatic disease can be achieved with as much as 30 Gy in 10 fractions and as little as 8 Gy delivered in a single fraction, depending on the clinical situation. The University of Washington has used single 8-Gy fractions to treat metastases in a variety of anatomic sites, and reported complete responses in $45 \%$ and local control in $77 \%$ of tumors treated. Brain metastases are rare with MCC; single case reports describe the use of whole-brain and stereotactic RT for treatment. ${ }^{42-44}$ As with other diseases, the approach used would typically depend on the extent of the intracranial and extracranial disease.

\section{RT and Immunotherapy}

Considerable advances have recently been made in harnessing antitumoral immunity for therapeutic gain. Immunosuppression increases the incidence of MCC and diminishes the prognosis of patients. Further, intratumoral immune response has been suggested to be prognostic in MCC. ${ }^{45}$ Randomized phase II trials in the metastatic setting have shown that pembrolizumab (anti-PD-1; overall response rate [ORR], 56\%) and avelumab (anti-PD-L1; ORR, $33 \%)$ are effective in MCC. ${ }^{46}$ Although durable responses are common, only a subset of patients experience clinical benefit. Considerable interest has been shown in improving outcomes through combination of immunotherapy with other agents, including RT. Preclinical models have indicated that RT is more effective with an intact adaptive immune system, and retrospective reviews have suggested that local control after RT is diminished in immunocompromised compared with immune-intact patients. ${ }^{47-49}$ Emerging evidence has not found increased toxicities when RT is administered concurrently with immune checkpoint blockades in melanoma. ${ }^{50}$ Adjuvant trials in MCC are ongoing and the hope is that information on the toxicity and efficacy of combination therapy will soon be available (ClinicalTrials. gov identifier: NCT03304639). Accordingly, a significant need exists to determine how best to combine immunotherapy and RT to improve outcomes in the adjuvant and metastatic settings.

\section{Conclusions}

The role of RT in the management of $\mathrm{MCC}$ is somewhat challenging to define because of the relative lack of data from prospective clinical trials. Nevertheless, a consistent finding in the literature is that RT is highly effective in providing locoregional control benefits in patients with MCC. Mostly single-institution experiences have confirmed that RT can be considered for the definitive, adjuvant, and palliative treatment of patients with MCC within a multidisciplinary framework. Similar to other disease sites, significant hope exists that future improvements in systemic control with immunotherapy will accentuate the importance of achieving locoregional control.

\section{References}

1. Bichakjian $C K$, Lowe $L$, Lao $C D$, et al. Merkel cell carcinoma: critical review with guidelines for multidisciplinary management. Cancer 2007;110:1-12.

2. Maksimovic S, Nakatani M, Baba Y, et al. Epidermal Merkel cells are mechanosensory cells that tune mammalian touch receptors. Nature 2014;509:617-621

3. Goldsmith L, Katz S, Gilchrest B, et al, eds. Fitzpatrick's Dermatology in General Medicine, 8th ed. New York, NY: McGraw-Hill; 2012.

4. Cheuk W, Kwan MY, Suster S, Chan J K. Immunostaining for thyroid transcription factor 1 and cytokeratin 20 aids the distinction of small cell carcinoma from Merkel cell carcinoma, but not pulmonary from extrapulmonary small cell carcinomas. Arch Pathol Lab Med 2001;125:228-231.

5. Feng $\mathrm{H}$, Shuda M, Chang Y, Moore PS. Clonal integration of a polyomavirus in human Merkel cell carcinoma. Science 2008;319:1096-1100.

6. Bhatia S, Afanasiev O, Nghiem P. Immunobiology of Merkel cell carcinoma: implications for immunotherapy of a polyomavirus-associated 
cancer. Curr Oncol Rep 2011;13:488-497.

7. Amin MB, Edge SB, Greene F, et al, eds. AJCC Cancer Staging Manual, 8th ed. New York, NY: Springer International Publishing; 2017.

8. Gupta SG, Wang LC, Penas PF, et al. Sentinel lymph node biopsy for evaluation and treatment of patients with Merkel cell carcinoma: the Dana-Farber experience and meta-analysis of the literature. Arch Dermatol 2006;142:685-690.

9. Bichakjian CK, Olencki T, Aasi SZ, et al. NCCN Clinical Practice Guidelines in Oncology: Merkel Cell Carcinoma. Version 1.2018. Accessed May 24, 2018. To view the most recent version of these guidelines, visit NCCN.org.

10. Iyer JG, Storer BE, Paulson KG, et al. Relationships among primary tumor size, number of involved nodes, and survival for 8044 cases of Merkel cell carcinoma. J Am Acad Dermatol 2014;70:637-643.

11. Meeuwissen JA, Bourne RG, Kearsley JH. The importance of postoperative radiation therapy in the treatment of Merkel cell carcinoma. Int J Radiat Oncol Biol Phys 1995;31:325-331.

12. Clark JR, Veness MJ, Gilbert R, et al. Merkel cell carcinoma of the head and neck: is adjuvant radiotherapy necessary? Head Neck 2007;29:249_ 257.

13. Gillenwater AM, Hessel AC, Morrison WH, et al. Merkel cell carcinoma of the head and neck: effect of surgical excision and radiation on recurrence and survival. Arch Otolaryngol Head Neck Surg 2001;127:149-154.

14. Jabbour J, Cumming R, Scolyer RA, et al. Merkel cell carcinoma: assessing the effect of wide local excision, lymph node dissection, and radiotherapy on recurrence and survival in early-stage disease-results from a review of 82 consecutive cases diagnosed between 1992 and 2004. Ann Surg Oncol 2007;14:1943-1952.

15. Morrison WH, Peters LJ, Silva EG, et al. The essential role of radiation therapy in securing locoregional control of Merkel cell carcinoma. Int J Radiat Oncol Biol Phys 1990;19:583-591.

16. Hitchcock CL, Bland KL, Laney RG III, et al. Neuroendocrine (Merkel cell) carcinoma of the skin. Its natural history, diagnosis, and treatment. Ann Surg 1988;207:201-207.

17. Lewis KG, Weinstock MA, Weaver AL, Otley CC. Adjuvant local irradiation for Merkel cell carcinoma. Arch Dermatol 2006;142:693-700.

18. Bhatia S, Storer BE, Iyer JG, et al. Adjuvant radiation therapy and chemotherapy in Merkel cell carcinoma: survival analyses of 6908 cases from the National Cancer Data Base. J Natl Cancer Inst 2016;108:pii:djw042.

19. Harrington $\mathrm{C}, \mathrm{K}$ wan W. Radiotherapy and conservative surgery in the locoregional management of Merkel cell carcinoma: the British Columbia Cancer Agency experience. Ann Surg Oncol 2016;23:573-578.

20. Frohm ML, Griffith KA, Harms KL, et al. Recurrence and survival in patients with Merkel cell carcinoma undergoing surgery without adjuvant radiation therapy to the primary site. JAMA Dermatol 2016;152:10011007.

21. Fields RC, Busam KJ, Chou JF, et al. Recurrence after complete resection and selective use of adjuvant therapy for stage I through III Merkel cell carcinoma. Cancer 2012;118:3311-3320.

22. Leonard JH, Ramsay JR, Kearsley JH, Birrell GW. Radiation sensitivity of Merkel cell carcinoma cell lines. Int J Radiat Oncol Biol Phys 1995;32:1401-1407

23. Mortier L, Mirabel X, Fournier C, et al. Radiotherapy alone for primary Merkel cell carcinoma. Arch Dermatol 2003;139:1587-1590.

24. Veness M, Foote M, Gebski V, Poulsen, M. The role of radiotherapy alone in patients with Merkel cell carcinoma: reporting the Australian experience of 43 patients. Int J Radiat Oncol Biol Phys 2010;78:703-709.

25. Veness M, Howle J. Radiotherapy alone in patients with Merkel cell carcinoma: the Westmead Hospital experience of 41 patients. Australas J Dermatol 2015;56:19-24.

26. Suntharalingam M, Rudholtz MS, Mendenhall WM, et al. Radiotherapy for merkel cell carcinoma of the skin of the head and neck. Head Neck 1995;17:96-101.

27. Poulsen M, Rischin D, Walpole E, et al. High-risk Merkel cell carcinoma of the skin treated with synchronous carboplatin/etoposide and radiation a Trans-Tasman Radiation Oncology Group study-TROG 96:07. J Clin Oncol 2003;21:4371-4376.

28. Jouary $\mathrm{T}$, Leyral C, Dreno B, et al. Adjuvant prophylactic regional radiotherapy versus observation in stage I Merkel cell carcinoma: a multicentric prospective randomized study. Ann Oncol 2012;23:10741080 .

29. Harrington C, Kwan W. Outcomes of Merkel cell carcinoma treated with radiotherapy without radical surgical excision. Ann Surg Oncol 2014;21:3401-3405.

30. Kitamura N, Tomita R, Yamamoto M, et al. Complete remission of Merkel cell carcinoma on the upper lip treated with radiation monotherapy and a literature review of Japanese cases. World J Surg Oncol 2015;13:152.

31. Gunaratne DA, Howle JR, Veness MJ. Definitive radiotherapy for Merkel cell carcinoma confers clinically meaningful in-field locoregional control: a review and analysis of the literature. J Am Acad Dermatol 2017;77:142148.e1.

32. Burmeister BH, Henderson MA, Ainslie J, et al. Adjuvant radiotherapy versus observation alone for patients at risk of lymph-node field relapse after therapeutic lymphadenectomy for melanoma: a randomised trial. Lancet Oncol 2012;13:589-597.

33. Schwartz JL, Griffith KA, Lowe L, et al. Features predicting sentinel lymph node positivity in Merkel cell carcinoma. J Clin Oncol 2011;29:10361041.

34. Burmeister BH, Mark Smithers B, Burmeister E, et al. A prospective phase II study of adjuvant postoperative radiation therapy following nodal surgery in malignant melanoma-Trans Tasman Radiation Oncology Group (TROG) study 96.06. Radiother Oncol 2006;81:136-142.

35. Fang LC, Lemos B, Douglas J, et al. Radiation monotherapy as regional treatment for lymph node-positive Merkel cell carcinoma. Cancer 2010;116:1783-1790.

36. George A, Girault S, Testard, et al. The impact of (18)F-FDG-PET/CT on Merkel cell carcinoma management: a retrospective study of 66 scans from a single institution. Nucl Med Commun 2014;35:282-290.

37. Mattes MD, Zhou Y, Berry SL, Barker CA. Dosimetric comparison of axilla and groin radiotherapy techniques for high-risk and locally advanced skin cancer. Radiat Oncol J 2016;34:145-155.

38. Foote M, Harvey J, Porceddu S, et al. Effect of radiotherapy dose and volume on relapse in Merkel cell cancer of the skin. Int J Radiat Oncol Biol Phys 2010;77:677-684.

39. Patel SA, Qureshi MM, Mak KS, et al. Impact of total radiotherapy dose on survival for head and neck Merkel cell carcinoma after resection. Head Neck 2017;39:1371-1377.

40. Patel SA, Qureshi MM, Sahni D, Truong MT. Identifying an optimal adjuvant radiotherapy dose for extremity and trunk Merkel cell carcinoma following resection: an analysis of the National Cancer Database. JAMA Dermatol 2017;153:1007-1014.

41. Poulsen MG, Rischin D, Porter I, et al. Does chemotherapy improve survival in high-risk stage I and II Merkel cell carcinoma of the skin? Int J Radiat Oncol Biol Phys 2006;64:114-119.

42. Jacob AT, Alexandru-Abrams D, Abrams EM, Lee JY. Stereotactic radiosurgery for Merkel cell carcinoma brain metastases. J Clin Neurosci 2015;22:1499-1502.

43. Ikawa F, Kiya K, Uozumi T, et al. Brain metastasis of Merkel cell carcinoma. Case report and review of the literature. Neurosurg Rev 1999;22:54-57.

44. Small KW, Rosenwasser GO, Alexander E III, et al. Presumed choroidal metastasis of Merkel cell carcinoma. Ann Ophthalmol 1990;22;187-190.

45. Ma JE, Brewer JD. Merkel cell carcinoma in immunosuppressed patients. Cancers (Basel) 2014;6:1328-1350.

46. Nghiem PT, Bhatia S, Lipson EJ, et al. PD-1 blockade with pembrolizumab in advanced Merkel-cell carcinoma. N Eng J Med 2016;374:2542-2552.

47. Nguyen $\mathrm{MH}$, Cook $\mathrm{M}$, Lachance $\mathrm{K}$, et al. Effect of immune status on outcomes and response to radiation among Merkel cell carcinoma patients [abstract]. Int J Radiat Oncol Biol Phys 2017;99(Suppl):Abstract 2857.

48. Deng L, Liang H, Burnette B, et al. Irradiation and anti-PD-L1 treatment synergistically promote antitumor immunity in mice. J Clin Invest 2014;124:687-695

49. Burnette BC, Liang $\mathrm{H}$, Lee $\mathrm{Y}$, et al. The efficacy of radiotherapy relies upon induction of type I interferon-dependent innate and adaptive immunity. Cancer Res 2011;71:2488-2496.

50. Barker CA, Postow MA, Khan SA, et al. Concurrent radiotherapy and ipilimumab immunotherapy for patients with melanoma. Cancer Immunol Res 2013;1:92-98. 